\title{
On Qian Zhongshu’s Huajing and Its Actual Practice
}

\author{
TANG Zhuoyi \\ Shanghai International Studies University, Shanghai, China
}

\begin{abstract}
Based on translation practice, Qian Zhongshu proposes the translation theory "Huajing”. The theory derives from Chinese traditional translation and revitalized with new ideas in Qian Zhongshu's translation practice. It brings together the essence of various translation theories and takes an important place in Chinese translation circles. The paper mainly focuses on "Huajing”'s theoretical basis and five core elements, and illustrates “Huajing”'s effectiveness with actual translation practice. The practice has proved that "Huajing" can help readers gain both understanding and sense of beauty of the source text, and also provide flexibility for translators in translation. Although standard of "Huajing” is not clear, it is still regarded as one of the greatest translation theories in China's translation circles.
\end{abstract}

Keywords: literary translation, Qian Zhongshu, “Huajing”

\section{Introduction}

Translation containing hidden echoes of the similar sense from source text is usually an ideal translation in China. As one of the most important traditional translation theories in China, "Huajing" (usually translated into "realm of transformation") describes what an ideal translation is like, posits a state that the successful translation is supposed to have reached, and which is out of bounds to poorer translations (Chan, 2004). The "Huajing” theory has long enjoyed prestige in Chinese translation circles and exerted a great influence on Chinese translation study and practice. This paper mainly focuses on the notion of "Huajing" and its practical enlightenment.

\section{Foundation of "Huajing"}

The term "Huajing" can be derived from Chinese translated version of Buddhist scriptures. Avatamsaka Sutra (《华严经》) mentioned the theory of "Satori” (realization to truth) and first talked about "Huajing”, regarding it as the highest standard of Buddhist theory. Hua can be both a verb and adjective with the sense of "transform" and "transformed" (Schulte, 2014). It refers to the aesthetic ideal in translation. Jing is denoted itself in Jingjie (境界), for the word can be inclusive of all the Chinese aesthetic significance and Buddhist sense. Jingjie (usually translated as "ideal state") was developed by Wang Guowei into "the center of aesthetic standard” (Rickett, 1977).

Qian Zhongshu gained his solid theoretical basis from both Chinese and western cultures. His "Huajing” is a variant version, being in common with Daoan's Wushiben (illustrates the difficulties and problems in translation and demands translators to make the format and expressions of their translation stick to the source text), Yanfu's three principles (faithfulness, comprehensiveness, and elegance), and Fu Lei’s spiritual 
resemblance theory (conveying the spirit of the source text and at the same time keeping fluency in target language).

\section{Notion of "Huajing"—Five Major Characters in "Huajing”}

The concept of "Huajing", is actually extended in meaning by Qian Zhongshu from Origin of Chinese Characters (《说文解字》). In Volume xii, under the heading “口”, “化” exists as the 26th character: “The word means translation. When the bird-catcher uses a live bird as a decoy, it is called 化” (Yu, 2007). Based on the concept given by Xu Shen, Qian Zhongshu made some modification and produced five main characters in “Huajing”: “yi” (译, translation), you (诱, guide), mei (媒, transit), e (讹, misrepresent), and hua (化, transform).

You (guide) and Mei (transit) are the functions of translation. The purpose of translation is to promote readers to observe and acquire knowledge the translated works, and to arouse readers' interests to appreciate and study the works.

E (misrepresent) is the inevitable losses in translation. Qian mentioned "the translated text always contains distortion and to some extent cannot fit the source text in meaning and in tone" (Yu, 2007). Pure mistranslation and creative mistranslation are two types of misrepresenting. The former is due to partial understanding of the source text as well as different culture backgrounds. Translators can even be disqualified in feeling and expressing the same spirit, thus misrepresenting exists. The latter, however, is the product of translator's subjective ideas. For example, Lin Shu would polish the source text when he thought it beautiful yet incomplete to make the language more specific, scenario livelier and description more beautiful. Qian Zhongshu appreciated translator's creative modification to the original text and thought it a way to provide true value of translation.

Hua (transform) is the core of "Huajing”. Qian Zhongshu regarded transform as the ideal of translation. He expounded his idea of "hua" as "the highest standard in literary translation, transforming a work from the language of one country into that of another” (Snell-Hornby, Jettmarová \& Kaindl, 1997 ). If translation can be done without betraying any evidence of source texts by virtue of divergences in language and speech habits, while at the same time preserving the sense of the original, then such a performance has attained "Huajing".

Hua contains two important parts: first, the language and speech habits of the target language must be followed in translation. Translator should acquire a correct understanding of the original text and extract the essence of it while not rigidly adhering to it or make it farfetched. The language transforms with the flavor and spirit of the source text unchanged. That's the core of hua.

Second, translation needs to achieve the ultimate art of translation within the language framework. Translator should pursue aesthetics in translation process (Cheung, 2014). Translation should not read like a translation but an aesthetic art. In Qian's opinion, translator should grasp the meaning with words forgotten (Zheng, 2010).

In general, guide, transit, misrepresenting, and transform are the core concept of "Huajing". In order to promote communications among different cultures and countries, guide and transit are the causes and functions of translation. Misrepresenting is the inevitable loss in translation, while it is also the bridge to realize the ideal status of translation. Transform is the ideal state of translation. 


\section{"Huajing” in Practice-A Case Study of China's Change: The Greatest Show on Earth}

The book China's Change: The Greatest Show on Earth was published in 2018, expressing Perman's philosophical ideas on China's changes, challenges, and snags.

My translation readership can be the general public who are interested in the both China and world affairs. The work can give readers an objective view of how foreign experts and writers think and analyze China's leaders. Thus in translation, I have to make adjustments to make the target text achieve "Huajing".

"Huajing”, at its core stage, is an aesthetic thought. As an ideology in translation, "Huajing” is difficult to achieve, and I need to apply various translation approaches and make full use of my knowledge to achieve "Huajing”. The examples below show how "E”, "Mei”, and "Hua” are adopted in translation.

"Mei"

SL: Xi borrowed the idea of "strategic configuration of power", a pearl of Chinese wisdom in Sun Tzu's Art of Martial Arts.

TL：习近平采用了权利战略配置的思维（即 “势”），这也是孙子兵法的精华。

Back translation: $\mathrm{Xi}$ adopted the thought of the strategic allocation of rights (the concept of "Shi" in Chinese), which is also the essence of Sun Tzu's Art of Martial Arts.

In order to keep a consistent translation style, I had to make a choice between domestication and foreignization. In the whole translation process, I expect my readers to gain the feeling of reading the exact source text and the author's views. Therefore, I adopt foreignization. "Strategic configuration of power" is actually the Chinese concept “Shi (势)". In order to keep the foreign language features, I literally translate it and add a functional equivalent to better explain it. The translation method can serve as the "Mei" concept in "Huajing": It connect the readers with the source text and can provide the readers with familiarity. In attracting readers to understand and explore foreigners' ideas about Chinese politics, this method can make a contribution.

"E"

(1) SL: Japan's government purchase of the Diaoyu/Senkaku Islands was a foreign policy gift for Xi.

$\mathrm{TL}$ : 日本政府购买钓鱼岛事件对习近平十分有利。

Back translation: Japan's government purchase of the Diaoyu Islands is to Xi's advantage.

The author undertakes a neutral position and an objective manner when facing the disputed issues. Therefore, he writes both "Diaoyu" and "Senkaku" in the text. However, Chinese readers take a firm stand in those issues, accepting only the "Diaoyu Islands". Different political positions lead to different translations. Therefore, in order to be consistent with the Chinese government and readers, I chose to omit "Senkaku" and only translate "Diaoyu Islands". Omitting belongs to "e" due to the change of the meaning and author's political position. However, in the text it is a creative translation since the aim of omitting is to satisfy government's political demand and in accord with people's common sense.

"Hua"

(1) SL: Xi has splashed the cash

TL: 习近平可以说是出手不凡。

Back translation: Xi Jinping's investment can be regarded as an extraordinary shot.

"Splash the cash" is a typical English idiom, implying rich men lavish money. In the source text, the idiom 
is used to demonstrate Xi's brave overseas investment. However, the expression has no equivalent in Chinese. I adopt the strategy "hua": deverbalize the source language and adapt it into the target language. Chinese four-word idioms are rich in meaning and strong in metrical sense, and can serve as a perfect choice in expressing the original meaning. Thus I use the idiom of similar meaning but dissimilar form “出手不凡”, meaning generous investment to replace the original idiom. The sense is kept and the meaning is accurately conveyed.

(2) SL: Asia’s other “little tigers” did the same more than 40 years ago.

TL：亚洲四小龙的其他成员国也在四十年前做了类似的事情。

Back translation: Other members of Asia’s four little Dragons have done something similar 40 years ago.

It is the standard metaphor that impedes the translation. The image of the dragon is different in east and west. In Western culture, the dragon represents evil and darkness with an image of sharp teeth and strong legs. However, in China, as well as in South Asia, the dragon is commonly the symbol of nobility, solemnness, holiness, and good fortune (Ku, 2012). It is the cultural difference that matters. English regards strong countries as "tiger" while Chinese uses "dragon". In order to make readers understand, I adopt the "hua" concept, changing the language form and image while keeping the language meaning and spirit. I reproduce the phrase to what readers familiar with. The idea of "hua" used here can provide readers with a familiar translation and a better understanding.

\section{Conclusions}

In the actual translation practice, we can find out the how efficient "Huajing" is. Adopting the concept of "yi”, “you”, "mei”, “e”, and "hua”, translator can provide readers with a target text which is rich in meaning and beautiful in language. Readers can therefore better understand the original text and accurately find out the core idea and spirit that the author wants to express. Qian Zhongshu's "Huajing” brings together the essence of various translation theories and takes an important place in Chinese translation circles. Though there are still places where improvements are needed, for example, Qian has not defined the "Huajing" through a critical discussion of his own work and has not provided the exact standard of transform; we still regard "Huajing" as the important theory in Chinese translation circles. Even today, "Huajing" still inspires translation theorists to explore the ideal realm as well as influencing translators.

\section{References}

Baker, M. (2011). In other words: A coursebook on translation. Abingdon-on-Thames: Routledge.

Chan, L. T. (2004). Twentieth-century Chinese translation theory: Modes, issues and debates. Amsterdam: John Benjamins Publishing.

Cheung, M. P. Y. (2014). 'Power and Ideology in Translation Research in Twentieth-Century China An Analysis of Three Seminal Works', Crosscultural Transgressions: Research Models in Translation: v. 2: Historical and Ideological Issues. Routledge, p. 144.

Snell-Hornby, M., Jettmarová, Z., \& Kaindl, K. (1997). Translation as intercultural communication: Selected papers from the EST Congress, Prague 1995. John Benjamins Publishing.

Yu, C. F. (2007). On Qian Zhongshu’s “theory of sublimity”. Perspectives: Studies in Translatology, 14(3), 214-229.

Zheng, X. (2010). 'A Study on Qian Zhongshu's Translation: Sublimation in Translation', Studies in Literature and Language. Canadian Research \& Development Center of Sciences and Cultures; Canadian..., 1(2), p. 70. 